\title{
Simplified Machine Diagnosis Techniques in Absolute Deterioration Factor by Using the 2nd Order AR Model
}

\author{
Kazuhiro Takeyasu ${ }^{1}$ \\ ${ }^{1}$ Tokoha University, Japan \\ Correspondence: Kazuhiro Takeyasu, Tokoha University, Japan.
}

Received: December 23, 2018

Accepted: January 11, 2019

Online Published: January 21, 2019

doi:10.5430/ijba.v10n1p61

URL: https://doi.org/10.5430/ijba.v10n1p61

\begin{abstract}
In order to make machine diagnosis, the method of calculating Kurtosis or Bicoherence was utilized. Calculating system parameter distance was also utilized applying time series data to Autoregressive (AR) model or Autoregressive Moving Average (ARMA) model.

In this paper, simplified calculation method of autocorrelation function is introduced and it is utilized for the 2nd order AR model identification. An absolute deterioration factor such as Bicoherence is also introduced. Furthermore, Mahalanobis' generalized distance is introduced by the relationship with system parameter distance. Three cases in which the rolling elements number is nine, twelve and sixteen are examined and compared. Machine diagnosis can be executed by this simplified calculation method of system parameter distance. Good results are obtained.
\end{abstract}

Keywords: AR model, diagnosis, Mahalanobis' generalized distance, absolute deterioration factor, rolling element

\section{Introduction}

In mass production firms such as steel making that have big equipments, sudden stops of production process by machine failure cause severe damages such as shortage of materials to the later process, delays to the due date and the increasing idling time.

To prevent these troubles, machine diagnosis techniques play important roles. So far, Time Based Maintenance (TBM) technique has constituted the main stream of the machine maintenance, which makes checks at previously fixed time. But it has a weak point that it makes checks at scheduled times without taking into account whether the parts are still keeping good conditions or not. On the other hand, Condition Based Maintenance (CBM) makes maintenance checks by watching the condition of machines. Therefore, if the parts are still keeping good condition beyond its expected life, the cost of maintenance may be saved because machines can used longer than planned. Therefore the use of CBM has become dominant. The latter one needs less cost of parts, less cost of maintenance and leads to lower failure ratio.

However, it is mandatory to catch a symptom of the failure as soon as possible if a transition from TBM to CBM is to be made. Many methods are developed and examined focusing on this subject. In this paper, we propose a method for the early detection of the failure on rotating machines that is the most common theme in machine failure detection field.

So far, many signal processing methods for machine diagnosis have been proposed (Bolleter, 1998; Hoffner, 1991). As for sensitive parameters, Kurtosis, Bicoherence, Impact Deterioration Factor (ID Factor) was examined (Yamazaki, 1977; Maekawa et al., 1997; Shao et al., 2001, Song et al., 1998; Takeyasu, 1989). Calculating system parameter distance was also utilized applying time series data to Autoregressive (AR) model or Autoregressive Moving Average (ARMA) model (Yamazaki, 1988).

In this paper, simplified calculation method of autocorrelation function is introduced and it is utilized for the 2nd order AR model identification. An absolute deterioration factor such as Bicoherence is also introduced. Furthermore, Mahalanobis' generalized distance is introduced by the relationship with system parameter distance. Three cases in which the rolling elements number is nine, twelve and sixteen are examined and compared.

In section 2, machine diagnosis techniques utilizing system parameter distance is exhibited. Parameter estimation method of AR model is stated in section 3. In section 4, simplified calculation method of autocorrelation function is introduced. Applying these methods, numerical examples are exhibited in section 5. In section 6, Mahalanobis' generalized distance is stated and we show the relationship with system parameter distance. Comparison of numerical calculation result is exhibited in section 7 . Section 8 is a summary. 


\section{Detection of Failure by System Parameter Distance}

In the analysis of time series data, Autoregressive (AR) model or Autoregressive Moving Average (ARMA) model are adopted frequently. In this paper, we adopt AR model, because it has a good estimation property (unbiased estimation) and is easy to identify.

Consider the $p$-th order AR model expressed as

$$
x_{n}+\sum_{i=1}^{p} a_{i} x_{n-i}=e_{n}
$$

Here

$\left\{x_{n}\right\}:$ Sample process of a stationary ergodic Gaussian process $x(t)$

$$
n=1,2,3, \cdots, N, \cdots
$$

$\left\{e_{n}\right\}$ : Gaussian white noise of mean 0, variance $\sigma_{e}^{2}$

Assume that (1) satisfies the stationarity condition.

Now, we calculate system parameter distance.

Let

$$
\begin{gathered}
\boldsymbol{a}=\left(\begin{array}{c}
a_{1} \\
a_{2} \\
\vdots \\
a_{p}
\end{array}\right) ; \text { system parameter under normal condition } \\
\hat{\boldsymbol{a}}=\left(\begin{array}{c}
\hat{a}_{1} \\
\hat{a}_{2} \\
\vdots \\
\hat{a}_{p}
\end{array}\right) ; \text { estimate of system parameter while abnormal condition proceeds }
\end{gathered}
$$

Then, following $Z$ is utilized as an evaluation function.

$$
Z=\frac{1}{\sqrt{\sum_{i=1}^{p}\left(a_{i}-\hat{a}_{i}\right)^{2}}+1}
$$

Watching the behavior of $Z$, we can judge the system becomes abnormal when $Z$ falls below the certain value (Figure 1). Then we can make failure detection. This evaluation function is an absolute deterioration factor such as Bicoherence. This is 1.0 when the system is under normal condition, and tends to be 0 when the system becomes abnormal.

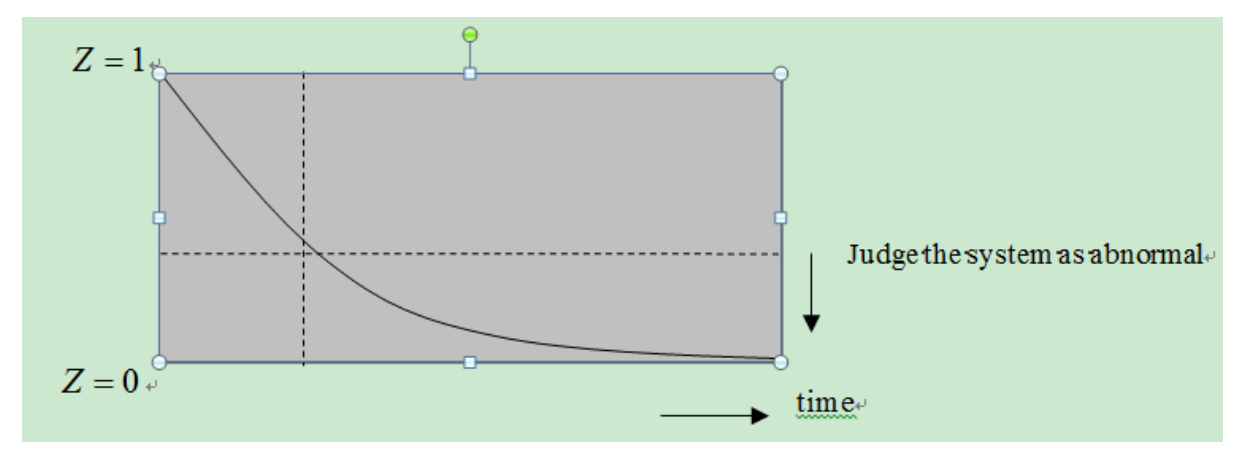

Figure 1. Detecting Abnormal Condition by the Transition of $Z$ 


\section{Estimation of System Parameter by Autoregressive Model}

In AR process, autocorrelation function satisfies the following equation (Tokumaru et al., 1982).

$$
R_{k}+a_{1} R_{k-1}+\cdots+a_{p} R_{k-p}=0 \quad(k>0)
$$

Here

$$
R_{k}=E\left[x_{n-k} x_{n}\right]
$$

Autocorrelation coefficient is as follows

$$
\rho_{k}=R_{k} / R_{0}
$$

Autocorrelation function satisfies the following equation.

$$
\rho_{k}+a_{1} \rho_{k-1}+\cdots+a_{p} \rho_{k-p}=0 \quad(k>0)
$$

Set $k=1,2, \cdots, p$, then we can get following equation from (8)

$$
\left.\begin{array}{c}
\rho_{1}+a_{1}+a_{2} \rho_{1}+\cdots+a_{p} \rho_{p-1}=0 \\
\rho_{2}+a_{1} \rho_{1}+a_{2}+\cdots+a_{p} \rho_{p-2}=0 \\
\vdots \\
\rho_{p}+a_{1} \rho_{p-1}+a_{2} \rho_{p-2}+\cdots+a_{p}=0
\end{array}\right\}
$$

This is the Yule-Walker equation that have unknown parameters $a_{1}, a_{2}, \cdots, a_{p}$.

$\boldsymbol{a}$ can be calculated by the following equation.

$$
\boldsymbol{a}=-\boldsymbol{H}^{-1} \boldsymbol{\rho}
$$

Where

$$
\begin{gathered}
\boldsymbol{H}=\left[\begin{array}{cccc}
1 & \rho_{1} & \cdots & \rho_{p-1} \\
\rho_{1} & 1 & \cdots & \rho_{p-2} \\
\vdots & \vdots & \ddots & \vdots \\
\rho_{p-1} & \rho_{p-2} & \cdots & 1
\end{array}\right] \\
\boldsymbol{\rho}=\left[\begin{array}{c}
\rho_{1} \\
\rho_{2} \\
\vdots \\
\rho_{p}
\end{array}\right]
\end{gathered}
$$

Autocorrelation function from $N\left\{x_{n} ; n=1,2, \cdots, N\right\}$ data is stated as

$$
\hat{R}_{k}=\frac{1}{N-k} \sum_{n=1}^{N-k} x_{n} x_{n+k}
$$


Let

$$
\hat{\rho}_{k}=\hat{R}_{k} / \hat{R}_{0}
$$

Then, $\hat{\boldsymbol{a}}$ is estimated as

$$
\hat{\boldsymbol{a}}=-\hat{\boldsymbol{H}}^{-1} \hat{\boldsymbol{\rho}}
$$

Where

$$
\begin{gathered}
\hat{\boldsymbol{H}}=\left[\begin{array}{cccc}
1 & \hat{\rho}_{1} & \cdots & \hat{\rho}_{p-1} \\
\hat{\rho}_{1} & 1 & \cdots & \hat{\rho}_{p-2} \\
\vdots & \vdots & \ddots & \vdots \\
\hat{\rho}_{p-1} & \hat{\rho}_{p-2} & \cdots & 1
\end{array}\right] \\
\hat{\boldsymbol{\rho}}=\left[\begin{array}{c}
\hat{\rho}_{1} \\
\hat{\rho}_{2} \\
\vdots \\
\hat{\rho}_{p}
\end{array}\right]
\end{gathered}
$$

Here, we examine the $2^{\text {nd }}$ order case $(p=2)$.

From (14), we get

$$
\begin{aligned}
{\left[\begin{array}{l}
\hat{a}_{1} \\
\hat{a}_{2}
\end{array}\right]=} & -\left[\begin{array}{cc}
1 & \hat{\rho}_{1} \\
\hat{\rho}_{1} & 1
\end{array}\right]^{-1}\left[\begin{array}{l}
\hat{\rho}_{1} \\
\hat{\rho}_{2}
\end{array}\right] \\
& =-\frac{1}{1-\hat{\rho}_{1}^{2}}\left(\begin{array}{c}
\hat{\rho}_{1}\left(1-\hat{\rho}_{2}\right) \\
\hat{\rho}_{2}-\hat{\rho}_{1}^{2}
\end{array}\right)
\end{aligned}
$$

Therefore

$$
\begin{aligned}
& \hat{a}_{1}=\frac{\hat{\rho}_{1}\left(\hat{\rho}_{2}-1\right)}{1-\hat{\rho}_{1}^{2}} \\
& \hat{a}_{2}=\frac{\hat{\rho}_{1}^{2}-\hat{\rho}_{2}}{1-\hat{\rho}_{1}^{2}}
\end{aligned}
$$

\section{Simplified Calculation Method of Autocorrelation Function}

Mean of $\left\{x_{i}\right\}$ is stated as follows.

$$
\bar{x}=\frac{1}{N} \sum_{i=1}^{N} x_{i}
$$

Variance of $\left\{x_{i}\right\}$ is stated as follows.

$$
\sigma^{2}=\frac{1}{N-1} \sum_{i=1}^{N}\left(x_{i}-\bar{x}\right)^{2}
$$


If $N$ is sufficiently large, variance of $\left\{x_{i}\right\}$ could be stated as

$$
\sigma^{2} \cong \frac{1}{N} \sum_{i=1}^{N}\left(x_{i}-\bar{x}\right)^{2}
$$

As $\left\{x_{i}\right\}$ is assumed to be stationary time series, we can assume $\bar{x}=0$ without loss of generality (Tokumaru et al., 1982).

Therefore, (13)can be re-stated as

$$
\hat{R}_{l}=\frac{1}{N-l} \sum_{i=1}^{N-l}\left(x_{i}-\bar{x}\right)\left(x_{i+l}-\bar{x}\right)
$$

That is to say, $0^{\text {th }}$ order autocorrelation function is nearly equal a variance.

When the number of failures on bearings or gears arise, the peak value arise cyclically. In the early stage of the defect, the peak signal usually appears clearly. Generally, defects will injure other bearings or gears by contacting the inner covering surface as time passes.

Assume that the peak signal which has $S$ times impact from normal signal arises in each $m$ times samplings. And also assume that mean and variance are same except for the case where a special peak signal arises.

Let of this case be $\bar{\sigma}^{2}$, then we get (Takeyasu et al., 2003-b)

$$
\begin{aligned}
& \bar{\sigma}^{2} \cong \frac{1}{N} \sum_{i=1}^{N}\left(x_{i}-\bar{x}\right)^{2} \\
\cong & \frac{N-\frac{N}{m}}{N} \sigma^{2}+\frac{\frac{N}{m}}{N} S^{2} \sigma^{2} \\
= & \left(1+\frac{S^{2}-1}{m}\right) \sigma^{2}
\end{aligned}
$$

For about autocorrelation function, let of this can be $\bar{R}_{i}(i=1,2, \cdots)$ in the same way. $0^{\text {th }}$ autocorrelation function is nearly equal variance, so we can get

$$
\bar{R}_{0} \cong \bar{\sigma}^{2} \cong\left(1+\frac{S^{2}-1}{m}\right) \sigma^{2}
$$

In the next, we examine the feature of autocorrelation function of $1^{\text {st }}$ order lag. From definition, $\bar{R}_{1}$ is stated as follows.

$$
\bar{R}_{1}=\frac{1}{N-1} \sum_{i=1}^{N-1} x_{i} x_{i+1}
$$

When the peak signal of $S$ times impact from normal signal arises in each $m$ times samplings, following $\wedge$ parts of $\left\{x_{i}\right\}$ may be considered to have peak value during the calculation of $\bar{R}_{1}$.

$$
1,2, \cdots, m-2, m-1, m, m+1, m+2, \cdots, 2 m-1,2 m, 2 m+1, \cdots
$$

From these, there arise following products.

1 product with peak value, $(m-1)$ products with ordinary level up to $m$

3 products with peak value, $(2 m-3)$ products with ordinary level up to $2 m$ 
$(2 k-1)$ products with peak value, $\{(m-2) k+1\}$ products with ordinary level up to $\mathrm{km}$

$\left(2 \cdot \frac{N-1}{m}-1\right)$ products with peak value, $\left\{(m-2) \cdot \frac{N-1}{m}+1\right\}$ products with ordinary level up to $N-1$

When $S$ is large, we make simplified calculation.

Suppose

$$
\begin{aligned}
& \bar{x}-\varepsilon<x_{i}<\bar{x}+\varepsilon \\
& \varepsilon>0,(i=1,2, \cdots, N)
\end{aligned}
$$

Then, we get following relations.

When $\bar{x}>0$

$$
\begin{aligned}
& \frac{1}{N-1}\left[S\left(2 \cdot \frac{N-1}{m}-1\right)+\left\{(m-2) \cdot \frac{N-1}{m}+1\right\}\right](\bar{x}-\varepsilon)^{2}<R_{1} \\
& <\frac{1}{N-1}\left[S\left(2 \cdot \frac{N-1}{m}-1\right)+\left\{(m-2) \cdot \frac{N-1}{m}+1\right\}\right](\bar{x}+\varepsilon)^{2}
\end{aligned}
$$

When $\bar{x}<0$

$$
\begin{gathered}
\frac{1}{N-1}\left[S\left(2 \cdot \frac{N-1}{m}-1\right)+\left\{(m-2) \cdot \frac{N-1}{m}+1\right\}\right](\bar{x}-\varepsilon)^{2}>R_{1} \\
>\frac{1}{N-1}\left[S\left(2 \cdot \frac{N-1}{m}-1\right)+\left\{(m-2) \cdot \frac{N-1}{m}+1\right\}\right](\bar{x}+\varepsilon)^{2}
\end{gathered}
$$

Suppose $\bar{x} \rightarrow 0$ and $\sigma^{2} \cong \varepsilon^{2}$, we get the following equation from (27).

$$
\begin{gathered}
\bar{\rho}_{1}=\frac{\bar{R}_{1}}{\bar{R}_{0}} \\
\cong \frac{1}{N-1}\left[S\left(2 \cdot \frac{N-1}{m}-1\right)+\left\{(m-2) \cdot \frac{N-1}{m}+1\right\}\right] \cdot \frac{1}{1+\frac{S^{2}-1}{m}} \\
=\left\{\left(\frac{2}{m}-\frac{1}{N-1}\right)(S-1)+1\right\} \cdot \frac{m}{m+S^{2}-1}
\end{gathered}
$$

(31) is the simplified calculation of coefficient of autocorrelation function of $1^{\text {st }}$ order lag. Under normal conditions, $\bar{\rho}_{1}=1$ when $S=1$. When $N \rightarrow \infty, S \rightarrow \infty, \bar{\rho}_{1} \rightarrow 0$ (Takeyasu et al., 2003-b).

Considering the case $S=2,4,6$, we obtain Table 1 from the calculation of (31) under the case $m=9,12,16$ and $N=100$. Here, $m$ is the number of rolling elements. 
Table $1 . \bar{\rho}_{1}$ by the variation of $S$

\begin{tabular}{cccc}
\hline & $S=2$ & $S=4$ & $S=6$ \\
\hline$m=9$ & 0.90909 & 0.61364 & 0.42149 \\
$m=12$ & 0.92525 & 0.65320 & 0.45519 \\
$m=16$ & 0.93886 & 0.69404 & 0.49396 \\
\hline
\end{tabular}

Next, we can get the following relation for $2^{\text {nd }}$ order lag in the same way.

$$
\bar{R}_{2}=\frac{1}{N-2} \sum_{i=1}^{N-2} x_{i} x_{i+2}
$$

Suppose $\bar{x} \rightarrow 0$ and $\sigma^{2} \cong \varepsilon^{2}$, we get the following equation from (27).

$$
\begin{gathered}
\bar{\rho}_{2}=\frac{\bar{R}_{2}}{\bar{R}_{0}} \\
\cong \frac{1}{N-2}\left[S\left(2 \cdot \frac{N-2}{m}-1\right)+\left\{(m-2) \cdot \frac{N-2}{m}+1\right\}\right] \cdot \frac{1}{1+\frac{S^{2}-1}{m}} \\
=\left\{\left(\frac{2}{m}-\frac{1}{N-2}\right)(S-1)+1\right\} \cdot \frac{m}{m+S^{2}-1}
\end{gathered}
$$

(33) is the simplified calculation of coefficient of autocorrelation function of $2^{\text {nd }}$ order lag. Under normal conditions, $\bar{\rho}_{2}=1$ when $S=1$. When $N \rightarrow \infty, S \rightarrow \infty, \bar{\rho}_{2} \rightarrow 0$ (Takeyasu et al., 2003-b).

Considering the case $S=2,4,6$, we obtain Table 2 from the calculation of (33) under the case $m=9,12,16$ and $N=100$.

Table 2. $\bar{\rho}_{2}$ by the variation of $S$

\begin{tabular}{cccc}
\hline & $S=2$ & $S=4$ & $S=6$ \\
\hline$m=9$ & 0.90901 & 0.61352 & 0.42138 \\
$m=12$ & 0.92517 & 0.65306 & 0.45506 \\
$m=16$ & 0.93878 & 0.69388 & 0.49380 \\
\hline
\end{tabular}

\section{System Parameter Distance by Simplified Calculation Method}

Now, we examine the $2^{\text {nd }}$ order case $(p=2)$.

When $S=1$, the system is considered to be under normal condition. Therefore we can get the following equation by (18), (19).

$$
\begin{aligned}
& a_{1}=\frac{\bar{\rho}_{1}\left(\bar{\rho}_{2}-1\right)}{1-\bar{\rho}_{1}^{2}} \\
& a_{2}=\frac{\bar{\rho}_{1}^{2}-\bar{\rho}_{2}}{1-\bar{\rho}_{1}^{2}}
\end{aligned}
$$

As 


$$
\begin{aligned}
& \bar{\rho}_{1}=1 \\
& \bar{\rho}_{2}=1
\end{aligned}
$$

Utilizing the following relation,

$$
\lim _{x \rightarrow 0} \frac{g(x)}{f(x)}=\lim _{x \rightarrow 0} \frac{g^{\prime}(x)}{f^{\prime}(x)}
$$

When

$$
\begin{aligned}
& \lim _{x \rightarrow 0} f(x)=0 \\
& \lim _{x \rightarrow 0} g(x)=0
\end{aligned}
$$

We obtain the following result.

$$
\begin{aligned}
& a_{1}=\lim _{\bar{\rho}_{1} \rightarrow 1, \bar{\rho}_{2} \rightarrow 1} \frac{\bar{\rho}_{2}-1}{-2 \bar{\rho}_{1}}=0 \\
& a_{2}=\lim _{\bar{\rho}_{1} \rightarrow 1, \bar{\rho}_{2} \rightarrow 1} \frac{2 \bar{\rho}_{1}}{-2 \bar{\rho}_{1}}=-1
\end{aligned}
$$

When $S=2,4,6$, we can get the following result by using the value in Table1, Table2 and calculating (34) (35).

Table 3. System parameter $\hat{a}_{i}$ by the variation of $S$

\begin{tabular}{cccc}
\hline$=9>$ & & & \\
\hline$S$ & 2 & 4 & 6 \\
\hline$\hat{a}_{1}$ & -0.4762 & -0.3804 & -0.2966 \\
$\hat{a}_{2}$ & -0.4757 & -0.3801 & -0.2964 \\
\hline
\end{tabular}

$<m=12>$

\begin{tabular}{crrr}
\hline$S$ & 2 & 4 & 6 \\
\hline$\hat{a}_{1}$ & -0.4811 & -0.3953 & -0.3129 \\
$\hat{a}_{2}$ & -0.4800 & -0.3949 & -0.3126 \\
\hline
\end{tabular}

\begin{tabular}{crrr}
$m=16>$ & & & \\
\hline$S$ & 2 & 4 & 6 \\
\hline$\hat{a}_{1}$ & -0.4849 & -0.4099 & -0.3307 \\
$\hat{a}_{2}$ & -0.4835 & -0.4094 & -0.3304 \\
\hline
\end{tabular}

In the case of $2^{\text {nd }}$ order, the evaluation function $Z$ is stated as follows from (4).

$$
Z=\frac{1}{\sqrt{\sum_{i=1}^{2}\left(a_{i}-\hat{a}_{i}\right)^{2}}+1}
$$

When $S=2,4,6$, we can get the following result by using the value in Table 4 and calculating (34), (35). 
Table 4. $Z$ by the variation of $S$

\begin{tabular}{ccrrr}
\hline & $S=1$ & $S=2$ & $S=4$ & $S=6$ \\
\hline$m=9$ & 1 & 0.5853 & 0.5789 & 0.5670 \\
$m=12$ & 1 & 0.5853 & 0.5804 & 0.5697 \\
$m=16$ & 1 & 0.5853 & 0.5818 & 0.5725 \\
\hline
\end{tabular}

As $S$ grows large, the evaluation function $Z$ becomes smaller.

Thus, we can utilize this index as machine diagnosis index.

\section{Mahalanobis' Generalized Distance}

System parameter distance is stated as follows.

$$
J=(\hat{\boldsymbol{a}}-\boldsymbol{a})^{\prime}(\hat{\boldsymbol{a}}-\boldsymbol{a})
$$

Where $\boldsymbol{a}$ is the system parameter under normal condition and $\hat{\boldsymbol{a}}$ is the estimated parameter while abnormal condition proceeds.

Thus, parameter distance $J$ (Euclidian distance) can be utilized as an index of machine diagnosis for detecting abnormal condition.

Next, characteristics of variance of forecasting error is examined. Forecasting error is stated as

$$
\begin{aligned}
F & =E\left[\hat{x}_{n+l}-x_{n+l}\right] \\
& =E\left[\hat{\boldsymbol{a}}^{\prime} \boldsymbol{x}_{n}-\boldsymbol{a}^{\prime} \boldsymbol{x}_{n}\right] \\
& =E\left[(\hat{\boldsymbol{a}}-\boldsymbol{a})^{\prime} \boldsymbol{x}_{n}\right]
\end{aligned}
$$

Where

$$
\boldsymbol{x}_{n}=\left(\begin{array}{l}
x_{n} \\
x_{n-1} \\
\vdots \\
x_{n-p}
\end{array}\right)
$$

Variance of forecasting error is expressed as

$$
\begin{aligned}
K & =\operatorname{Var}[F]=E\left[\left(\hat{x}_{n+l}-x_{n+l}\right)^{2}\right] \\
& =E\left[\left\{(\hat{\boldsymbol{a}}-\boldsymbol{a})^{\prime} \boldsymbol{x}_{n}\right\}\left\{(\hat{\boldsymbol{a}}-\boldsymbol{a})^{\prime} \boldsymbol{x}_{n}\right\}^{\prime}\right] \\
& =E\left[(\hat{\boldsymbol{a}}-\boldsymbol{a})^{\prime} \boldsymbol{x}_{n} \boldsymbol{x}_{n}^{\prime}(\hat{\boldsymbol{a}}-\boldsymbol{a})\right] \\
& =(\hat{\boldsymbol{a}}-\boldsymbol{a})^{\prime} E\left[\boldsymbol{x}_{n} \boldsymbol{x}_{n}^{\prime}\right](\hat{\boldsymbol{a}}-\boldsymbol{a})
\end{aligned}
$$




$$
\begin{aligned}
& =(\hat{\boldsymbol{a}}-\boldsymbol{a})^{\prime}\left(\begin{array}{cccc}
R_{0} & R_{1} & \cdots & R_{p-1} \\
R_{1} & R_{0} & \cdots & R_{p-2} \\
\vdots & \vdots & \ddots & \vdots \\
R_{p-1} & R_{p-2} & & R_{0}
\end{array}\right)(\hat{\boldsymbol{a}}-\boldsymbol{a}) \\
= & (\hat{\boldsymbol{a}}-\boldsymbol{a})^{\prime} \boldsymbol{R}(\hat{\boldsymbol{a}}-\boldsymbol{a})
\end{aligned}
$$

Where

$R_{i}$ : autocorrelation function of the original series $\operatorname{signal}(i=0, \cdots, p-1)$

$\boldsymbol{R}$ : matrix of autocorrelation function

That is to say that variance of forecasting error means system parameter distance with the weight of $\boldsymbol{R}$. By the way, Mahalanobis' generalized distance $D_{0}^{2}$ is stated as

$$
D_{0}^{2}=\left(\hat{\boldsymbol{a}}_{\boldsymbol{i} \boldsymbol{k}}-\boldsymbol{a}\right)^{\prime} \Sigma^{-1}\left(\hat{\boldsymbol{a}}_{\boldsymbol{i k}}-\boldsymbol{a}\right)
$$

Where

$$
\begin{gathered}
\boldsymbol{\Sigma}=\operatorname{Var}[\hat{\boldsymbol{a}}] \\
=E\left[(\hat{\boldsymbol{a}}-\boldsymbol{a})(\hat{\boldsymbol{a}}-\boldsymbol{a})^{\prime}\right] \\
=\left(\boldsymbol{\sigma}_{k l}\right) \\
\sigma_{k l}=\frac{1}{N_{0}} \sum_{i=1}^{N_{0}}\left(\hat{a}_{i k}-a_{k}\right)\left(\hat{a}_{i l}-a_{l}\right)
\end{gathered}
$$

Under the normal condition,

$$
E[\hat{a}]=\boldsymbol{a}
$$

Therefore the variance of $\hat{\boldsymbol{a}}$ is as follows.

$$
E\left[(\hat{\boldsymbol{a}}-E[\hat{\boldsymbol{a}}])(\hat{\boldsymbol{a}}-E[\hat{\boldsymbol{a}}])^{\prime}\right]=E\left[(\hat{\boldsymbol{a}}-\boldsymbol{a})(\hat{\boldsymbol{a}}-\boldsymbol{a})^{\prime}\right]
$$

On the other hand (Tokumaru et al., 1982),

$$
E\left[(\hat{\boldsymbol{a}}-\boldsymbol{a})(\hat{\boldsymbol{a}}-\boldsymbol{a})^{\prime}\right]=\frac{1}{N} \sigma_{e}^{2} \boldsymbol{R}^{-1}
$$

Comparing (41) with (44), we can get following equation.

Then $D_{0}^{2}$ can be re-stated as

$$
\Sigma=\frac{\sigma_{e}^{2}}{N} \boldsymbol{R}^{-1}
$$

$$
\begin{aligned}
D_{0}^{2}= & (\hat{\boldsymbol{a}}-\boldsymbol{a})^{\prime} \Sigma^{-1}(\hat{\boldsymbol{a}}-\boldsymbol{a}) \\
& =(\hat{\boldsymbol{a}}-\boldsymbol{a})^{\prime} \frac{N}{\sigma_{e}^{2}} \boldsymbol{R}(\hat{\boldsymbol{a}}-\boldsymbol{a})
\end{aligned}
$$


As is stated above, Mahalanobis' generalized distance means a system parameter distance with the weight of $N / \sigma_{e}^{2} \boldsymbol{R}$ and variance of forecasting error is itself a system parameter distance with the weight of $\boldsymbol{R}$ which means that it is $\sigma_{e}^{2} / N$ times of Mahalanobis' generalized distance.

\section{Comparison of the Both Methods}

(1) Calculation of the variance of forecasting error

Here, we calculate the variance of forecasting error and compare the results of (37) and (39).

As $x(t)$ is assumed to be the stationary ergodic Gaussian process, we can assume mean is 0 , variance is 1 without loss of generality (Tokumaru et al., 1987).

Therefore we can get the following equations.

$$
\begin{aligned}
& R_{0}=\sigma^{2}=1 \\
& R_{k}=\rho_{k} R_{0}=\rho_{k}
\end{aligned}
$$

It means

$$
\rho_{0}=1
$$

$K$ is calculated using $\rho_{k}, a_{k}$ obtained in 5 .

Here, we examine the $2^{\text {nd }}$ order case.

$$
K=\left(\begin{array}{l}
\hat{a}_{1}-a_{1} \\
\hat{a}_{2}-a_{2}
\end{array}\right)^{\prime}\left(\begin{array}{ll}
R_{0} & R_{1} \\
R_{1} & R_{0}
\end{array}\right)\left(\begin{array}{l}
\hat{a}_{1}-a_{1} \\
\hat{a}_{2}-a_{2}
\end{array}\right)
$$

The calculation result is exhibited in Table 5.

Table $5 . K$ by the variation of $S$

\begin{tabular}{lccc}
\hline & $S=2$ & $S=4$ & $S=6$ \\
\hline$m=9$ & 0.0477 & 0.2396 & 0.4071 \\
$m=12$ & 0.0389 & 0.2099 & 0.3746 \\
$m=16$ & 0.0316 & 0.1808 & 0.3390 \\
\hline
\end{tabular}

Now, we define $W$ as follows.

$$
W=\frac{1}{K+1}
$$

When $S=2,4,6$, we can get the following result by using the value in Table 5 .

Table $6 . W$ by the variation of $S$

\begin{tabular}{ccccc}
\hline & $S=1$ & $S=2$ & $S=4$ & $S=6$ \\
\hline$m=9$ & 1 & 0.9544 & 0.8067 & 0.7107 \\
$m=12$ & 1 & 0.9626 & 0.8265 & 0.7275 \\
$m=16$ & 1 & 0.9693 & 0.8469 & 0.7468 \\
\hline
\end{tabular}

As $S$ grows larger, the evaluation function $W$ becomes smaller.

Thus, we can utilize this index as machine diagnosis index (Table 6).

Comparing $Z$ with $W, Z$ is apparently sensitive.

The value of $W$ goes down smoothly as $S$ grows large compared with those of $Z$. 
Therefore it is required to choose the suitable one based upon the condition where the users are in.

\section{Conclusions}

In order to make machine diagnosis, the method of calculating Kurtosis or Bicoherence was utilized in the past. Calculating system parameter distance was also utilized applying time series data to Autoregressive (AR) model or Autoregressive Moving Average (ARMA) model.

In this paper, simplified calculation method of autocorrelation function was introduced and it was utilized for the 2nd order AR model identification. An absolute deterioration factor such as Bicoherence was also introduced. Furthermore, Mahalanobis' generalized distance was introduced by the relationship with system parameter distance. Three cases in which the rolling elements number was nine, twelve and sixteen were examined and compared. Machine diagnosis could be executed by this simplified calculation method of system parameter distance.

Proposed method proved to be a practical index for machine diagnosis by numerical examples.

The effectiveness of this method should be examined in various cases.

\section{References}

Bolleter, U. (1988). Blade Passage Tones of Centrifugal Pumps. Vibration, 4(3), 8-13.

Hino, M. (1977). Spectrum Analysis (in Japanese). Asakura shoten Publishing.

Maekawa, K., Nakajima, S., \& Toyoda, T. (1997). New Severity Index for Failures of Machine Elements by Impact Vibration (in Japanese). J.Sope Japan, 9(3), 163-168.

Noda. (1987). Diagnosis Method for a Bearing (in Japanese). NSK Tec. J., (647), 33-38.

Shao, Y., Nezu, K., Matsuura, T., Hasegawa, Y., \& Kansawa, N. (2001). Bearing Fault Diagnosis Using an Adaptive Filter (in Japanese). J.Sope Japan, 12(3), 71-77.

Song, J.W., Tin, H., \& Toyoda, T. (1998). Diagnosis Method for a Gear Equipment by Sequential Fuggy Neural Network (in Japanese). J. Sope Japan, 10(1), 15-20.

Takeyasu, K., \& Higuchi, Y. (2006). Simplified Machine Diagnosis Techniques by Impact Vibration using Higher Order Cumulants of Absolute Deterioration Factor, Osaka Prefecture University. Journal of Economics, Business and Law, 8, 25-39.

Takeyasu, K., \& Ishii, Y. (2005). System Parameter Distance for Machine Diagnosis. IFORS.

Takeyasu, K., Amemiya, T., Ino, K., \& Masuda, S. (2003-a). Machine Diagnosis Techniques by Simplified Calculation Method. IEMS, 2(1), 1-8.

Takeyasu, K., Amemiya, T., Ino, K., \& Masuda, S. (2003-b). Simplified Machine Diagnosis Techniques by Impact Vibration-Deterioration Factor of Autocorrelation Function Type (In Japanese). J.SOPE Japan, 15(3), 138-143.

Takeysu, K. (1989). Watching Method of Circulation Moving Object (in Japanese). Certified Patent by Japanese Patent Angency.

Tokumaru, H., Soeda, T., Nakamizo, T., \& Akizuki, K. (1982). Measurement and Calculation (in Japanese). Baifukan Publishing.

Yamazaki, H. (1977). Failure Detection and Prediction (in Japanese). Kogyo Chosakai Publishing. 